\title{
Antibiotics prescription in the elderly across different clinical areas; A real-life clinical audit
}

\author{
Authors: Khaled Madi; Joyce Luk; Seline Sutton; Amr Elkammash; Sathyabama \\ Loganathan \\ The Royal Bournemouth Hospital, University Hospitals Dorset NHS Foundation Trust, Bournemouth, \\ UK \\ Corresponding author: Dr Khaled Madi. Email: Khaled.madi@uhd.nhs.uk.
}

\begin{abstract}
:
Ageing of the population represents a great challenge to the national health system (NHS) in the UK. Patients in this age group have multiple comorbidities and use polypharmacy. They also have alterations in the absorption, metabolism and excretion of drugs. These factors make them susceptible to developing side effects and drug interactions on using antibiotics.

Our team audited the safety of antibiotic prescription in elderly patients (above 85 years of age) in regards to the selection of the appropriate antibiotics, and the documentation of the indication and duration of them. We also compared the prescription process during working hours and out of hours. We recruited 20 patients from each of the three departments involved in their care: The Old people admission unit (OPAU), the geriatric wards and other hospital wards. Our minimum compliance rate was $95 \%$ in each of the studied parameters. We spotted a significant compromise in the antibiotic prescription on non-geriatric wards and out of hours. This goes with the concerns raised by other studies. We recommend continuous education and the setting up of campaigns to increase the awareness of the medical staff on this aspect.
\end{abstract}

Keywords: audit, antibiotics, geriatric, elderly, out of hours, NHS, UK.

\section{Problem:}

The percentage of the elderly (above 85 years of age) in the British population is growing continuously. This sector usually has complex medical needs. ${ }^{1}$ They use a lot of medications with different pharmacokinetic properties. This leaves them at risk of developing significant side effects when being started on antibiotics.

Bournemouth lies on the southern coast of the UK. It is a town where British people prefer to spend their post-retirement life in. The Royal Bournemouth Hospital forms the bigger part of the University Hospitals Dorset NHS Foundation Trust. It serves a large number of elderly patients. It was highly important to check the safety of antibiotic prescription in this vulnerable age group. Therefore, our group carried out a clinical audit to monitor antibiotic prescription indication and duration, and compare it to the local guidelines. 


\section{Background:}

The elderly have alterations in drug absorption, metabolism and excretion. They have multiple comorbidities and use polypharmacy. They are at risk of drug accumulation and the manifestation of side effects. ${ }^{2}$ Researchers found that antibiotics safe prescription according to the guidelines was undermined in this age group. They found this safety even worse on increasing the number of antibiotics prescribed and patients' comorbidities. ${ }^{3}$

\section{Methods:}

We studied 20 patients aged above 85 years from each of the departments involved in the treatment of the elderly ( Old people admission unit ( OPAU), geriatric wards, and other hospitals wards), with a total number of 60 patients. Geriatric outliers are geriatric patients who are treated on non-geriatric wards due to a shortage of beds. We used the medical records and the drug charts to check the appropriateness of the chosen antibiotic, antibiotic prescription indication and duration. We also compared the prescription during working hours versus out of hours $(\mathrm{OOH})$. Our minimum acceptable compliance rate for the appropriateness of antibiotics was $95 \%$. Our minimum acceptable compliance rate for antibiotic indication and duration documentation was $95 \%$, both during working hours and out of hours. We considered the local hospital antibiotic guidelines as our reference for the selection of antibiotics.

\section{Results:}

Data from the OPAU showed that the appropriate antibiotic was chosen in $94 \%$ of cases. The indication was documented in all patients. The duration was documented in only $62 \%$ during working hours and $69 \%$ out of hours. Out of hours prescription was reviewed on day ward round in $67 \%$ of cases only. (fig 1 )

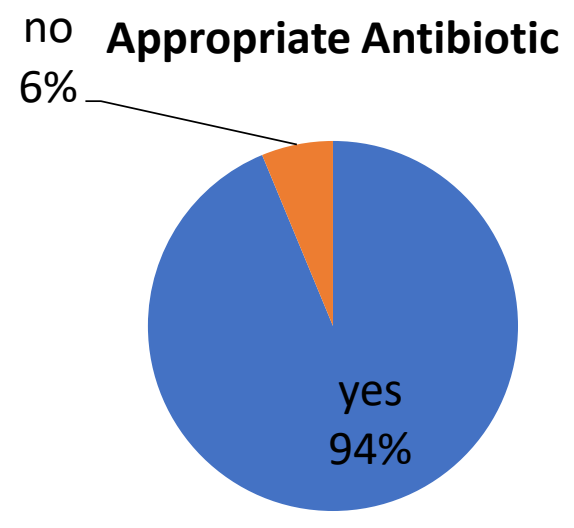

\section{Indication documented}

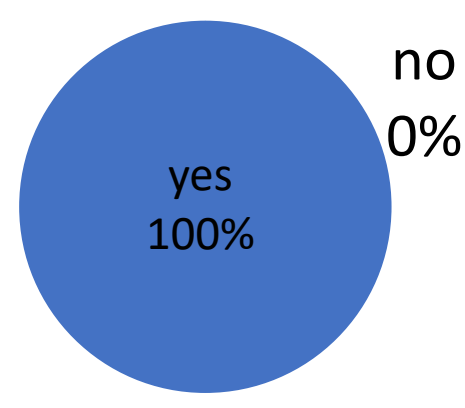


Duration documented

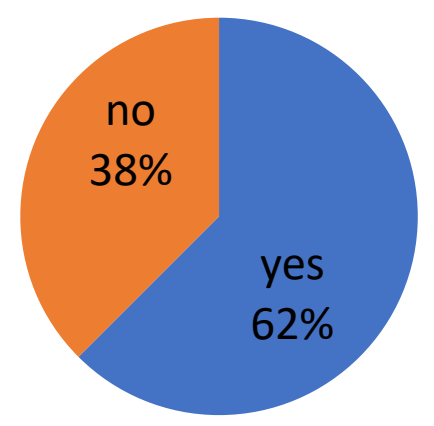

$\mathrm{OOH}$ prescription

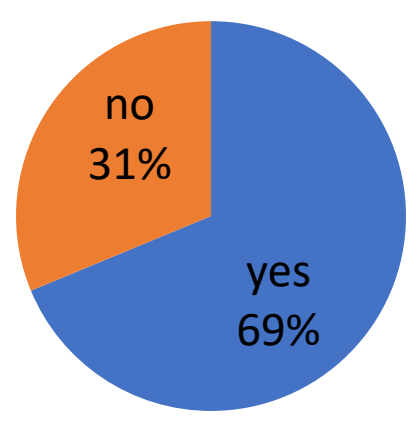

Figure 1: Data from OPAU

In the geriatric wards, doctors chose the appropriate antibiotic in all the patients (100\%). The indication was documented in $96 \%$ of the case, and the duration was documented in only $56 \%$. Out of hours documentation of indication and duration was even less. (36\%) (fig 2)

\section{Appropriate antibiotics}

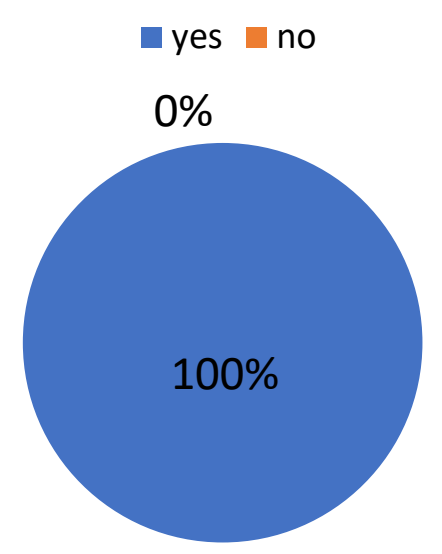

\section{Indication documented}

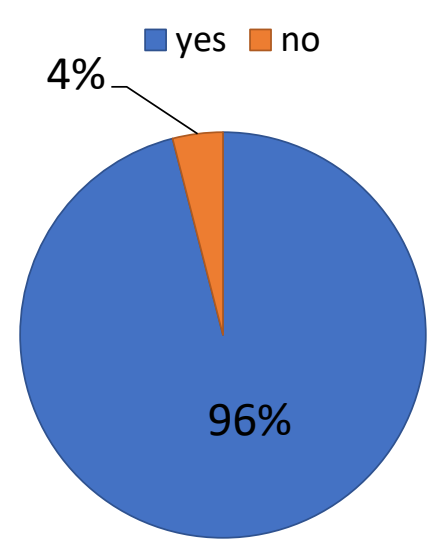




\section{Duration documented}

$\square$ yes no

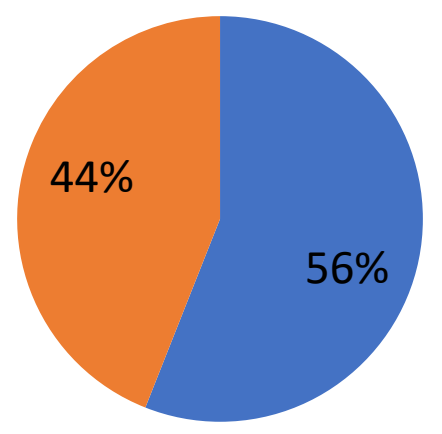

\section{$\mathrm{OOH}$ prescription}

ayes no

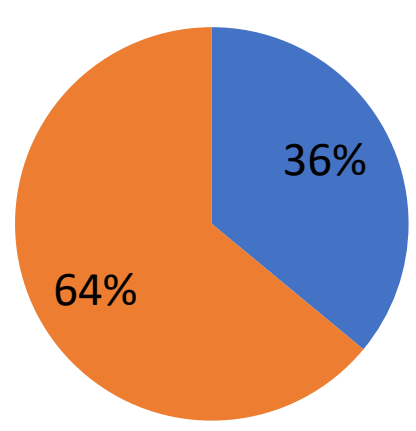

Figure 2: Data from the geriatric wards

In the case of geriatric outliers, doctors prescribed the appropriate antibiotic in all patients $(100 \%)$. However, the rates of documentation of the indication and duration of the antibiotic were quite low ( $74 \%$ and $52 \%$ respectively). These were documented in $52 \%$ of the patient out of hours. (fig 3)

Appropriate antibiotics

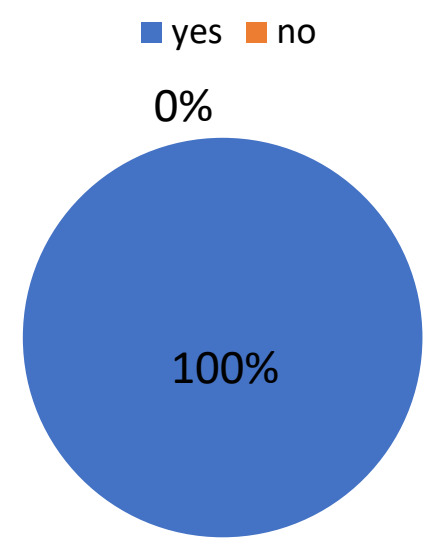

\section{Indication documented}

घyes no

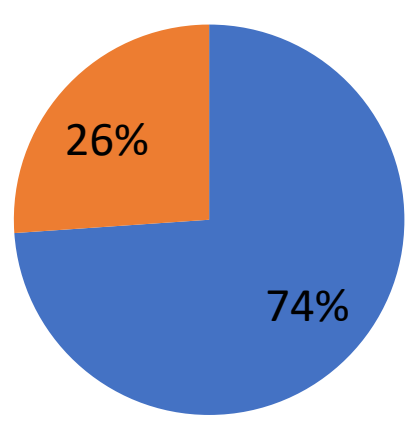




\section{Duration documented}

\section{$\mathrm{OOH}$ prescription}
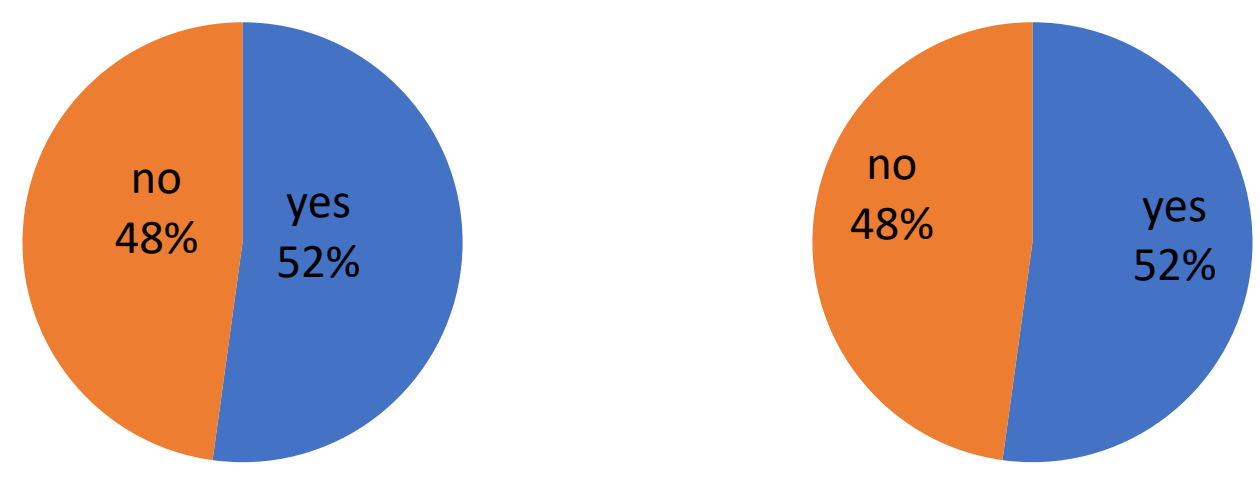

Figure 3: data of geriatric outliers

\section{Discussion:}

Our audit uncovered a defect in the antibiotic prescription in elderly patients. This defect was more remarkable on non-geriatric medical wards and in out of hours prescriptions. This can be explained by the lower staff number and the higher amount of distraction in these contexts.

Our findings agree with the concerns raised on the admission of medical patients to inappropriate wards. Studies showed that this was associated with a higher rate of patient dissatisfaction. ${ }^{4}$ It was also associated with a longer hospital stay. Such studies did not show an increase in morbidity and mortality of outlier patients. ${ }^{5,6}$

Out of hours service is a big challenge in the NHS. A lot of effort is being made to improve the quality of service in these hours. ${ }^{7}$ Our audit highlights an impairment in antibiotic prescription during these hours. Medical staff working on evening and night shifts should be more cautious to avoid compromise of patient safety.

\section{Conclusion:}

Antibiotic prescription to elderly patients out of hours and on non-geriatric wards needs special attention from the responsible medical staff and the hospitals' management boards. Our audit team propose that continuous medical education and awareness campaigns can help to get over this problem.

\section{References:}

[1] Kingston A, Comas-Herrera A, Jagger C. Forecasting the care needs of the older population in England over the next 20 years: estimates from the Population Ageing and Care Simulation (PACSim) modelling study. Lancet Public Heal 2018; 3: e447. 
[2] Miller LW, Guglin M. Patient selection for ventricular assist devices: A moving target. Journal of the American College of Cardiology 2013; 61: 1209-1221.

[3] Dylis A, Boureau AS, Coutant A, et al. Antibiotics prescription and guidelines adherence in elderly: Impact of the comorbidities. BMC Geriatr 2019; 19: 1-6.

[4] Goulding L, Adamson J, Watt I, et al. Lost in hospital: a qualitative interview study that explores the perceptions of NHS inpatients who spent time on clinically inappropriate hospital wards. Heal Expect 2015; 18: 982-994.

[5] Stylianou N, Fackrell R, Vasilakis C. Are medical outliers associated with worse patient outcomes? A retrospective study within a regional NHS hospital using routine data. BMJ Open; 7. Epub ahead of print 1 May 2017. DOI: 10.1136/BMJOPEN-2016015676.

[6] Alameda C, Suárez C. Clinical outcomes in medical outliers admitted to hospital with heart failure. Eur J Intern Med 2009; 20: 764-767.

[7] Beckett DJ, Gordon CF, Paterson R, et al. Improvement in out-of-hours outcomes following the implementation of Hospital at Night. QJM An Int J Med 2009; 102: 539546. 